\title{
TAPS HEXAGONAL EN EL DESARROLLO DEL PENSAMIENTO LÓGICO
}

\section{TAPS HEXAGONAL IN THE LOGICAL THOUGHT'S DEVELOPMENT}

\section{RESUMEN}

\author{
Ivan Rojas
}

El juego consiste en armar los taps hexagonal con números y usar los signos matemáticos de las operaciones básicas, intermedias y avanzadas según el nivel de que se encuentre. El alumno debe tratar de formar 5 a más ecuaciones en poco tiempo con los taps hexagonales. En el presente proyecto hemos elegido los hexagonales que se adaptan mejor a nuestros intereses y abarcar los ámbitos de las matemáticas ya que hay trabajos que no se dedican a los niveles de transferencia y las dimensiones sociales.

Palabra Clave: Juegos Matemáticos, Matemática Recreativa.

\begin{abstract}
The game consists of arming the taps hex numbers and using mathematical signs of the core operations, intermediate and advanced levels as it is. The student must try to form 5 to more equations in a short time with taps hexagonal. In this project we chose the hexagonal that are better suited to our interests and cover the fields of mathematics as there are jobs that are not dedicated to the levels of transfer and the social dimensions.
\end{abstract}

Key word: Mathematical Games, Mathematical Recreativa

\section{INTRODUCCIÓN}

Los taps hexagonal son instrumentos de juegos muy utilizados hoy en día y son de preferencia en juegos grupales de alumnos, en los recreos o juegos de amigos en el barrio.

El juego consiste en armar los taps hexagonal con números y usar los signos matemáticos de las operaciones básicas, intermedias y avanzadas según el nivel de que se encuentre. El niño debe tratar de formar 5 a más ecuaciones en poco tiempo con los taps hexagonales.

Los taps los hay circulares, hexagonal, triangulares en el presente trabajo hemos elegido los hexagonales que se adaptan mejor a nuestros intereses y abarcar los ámbitos de las matemáticas ya que hay trabajos que no se dedican a los niveles de transferencia y las dimensiones sociales.

En este sentido, la escuela, tiene como obligación crear las condiciones para el desarrollo del niño. $Y$ esas, se hallan plasmadas cuando, la escuela selecciona el contenido de la actividad (entendiendo como contenido las condiciones de vida material) y la forma (la conciencia social), ejercitar y practicar constantemente las acciones que el docente le asigna para lograr su consolidación, reforzar los aprendizajes adquiridos, formar hábitos, cumplir las tareas asignadas, desarrollar la educación general de la comunidad y dar nuevas exigencias a los alumnos, hasta que finalmente sepa transformar su realidad.

\section{JUSTIFICACIÓN}

Se pretende dar a conocer los taps hexagonal como materiales de tipo manipulativo, con los que se puede trabajar la mayoría de los contenidos correspondientes a los diferentes ámbitos de la matemática para alumnos en edades comprendidas entre 7 a más años.
Estos materiales se han elaborado teniendo en cuenta algunas partes de unas estructuras hexagonales tomadas de los panales de las abejas como base y que son muy adecuados para el uso en aula la precisión al momento de unirlos. Se proponen unos modelos de actividades a desarrollar con estos materiales, que pueden servir de ejemplo para crear otras similares.

Y para solucionar este problema, el presente estudio, tiene como propósito alcanzar a los alumnos del nivel primario una propuesta de cómo los taps hexagonal a partir del adecuado uso en los diferentes ámbitos de la matemática (aritmética, geometría, algebra); lo cual permitirá desarrollar el pensamiento lógico de los alumnos del colegio mediante el juego.

\section{METODOLOGÍA}

Como método general se utilizará el Método Científico que permitirá la observación y formulación del problema de la influencia de los taps hexagonal en el desarrollo del pensamiento lógico.

Luego de ello, ayudará a la construcción de la hipótesis como respuesta a la interrogante de investigación, la operacionalización teórica de las variables, la confrontación y análisis estadístico de los resultados y la prueba de hipótesis.

En todo este método también se aplicarán métodos lógicos como la inducción y la deducción cuyo principio en el primero será a partir de observaciones aisladas y de la abstracción cuando se trata de la segunda.

Estrategias para la aplicación, Recolección y Procesamiento de los datos obtenidos

- Evaluación de entrada a los alumnos.

- Clasificación y diseño de los alumnos en grupos homogéneos y heterogéneos.

\footnotetext{
${ }^{1}$ Santiago, L. y Gonzalo, T. (2006). "Abejas y geometría"
} 
- Aplicación de los taps hexagonal para formar ecuaciones.

- Evaluación después del experimento.

\section{Materiales y Equipos de Investigación}

- Taps hexagonal: $60-100$

- 1 tijera

- Plumón (negro - rojo - azul)

- Lápices de colores

- Cartulina o papel bond de 80 grs. 4 hojas

- Papel bond de 80 grs. (80 hojas)

- Cinta de embalaje - masking tape.

- Recipiente de plástico con tapa 04

- Cámara fotográfica.

\section{Procedimiento}

1. Se cortan las cartulinas en forma de hexágonos (taps) de lo contrario se tienen los taps normales (circulares) $y$ se procede a cortar los extremos formando hexágonos.

2. Se escriben los números en los taps hexagonal ya formados.

3. Los taps deben estar en orden número formando grupos de 10 unidades cada uno es decir: del 0 al 10; 11 al $20, \ldots$ de manera que facilite su ubicación.

4. Forrarlos con la cita masking tape para no deteriorarlos.

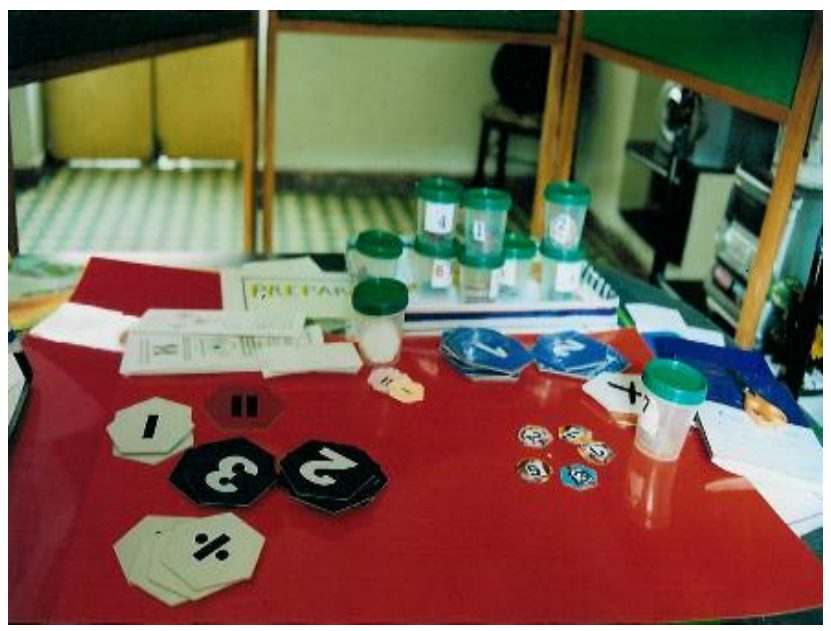

Figura 01. Materiales para elaborar taps.

\section{Reglas del Juego:}

1. El juego es entre dos o más participantes, el objetivo del juego es obtener la mayoría de puntos.

2. A cada jugador se le reparte una tarjeta de puntaje.

3. Para obtener puntos tienes que hacer ecuaciones matemáticas válidas.

4. Para cada ecuación que se crea cada pieza vale un punto, ejemplo: $3+4=7$, la ecuación vale 5 puntos, porque se usan 5 piezas.
TAPS HEXAGONAL

TARJETA DE PUNTAJE

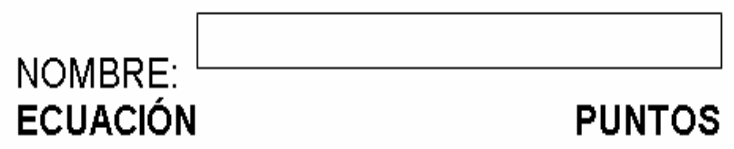

$3+4=7$

5

Figura 02. Tarjeta de puntaje.

Inicio del juego.

1. Encontrar la pieza "roja" y ubicarla en el cuadro del tablero (tales como una mesa) esparcir las piezas de los taps hexagonal volteadas a la pieza "=".

2. El jugador con el mayor número empieza usando los signos matemáticos,,$+- \times, \div$. Ubica las piezas al revés, en el grupo de las piezas volteadas; el juego continúa en dirección horario u antihorario.

3. Cada jugador escoge una pieza de los demás jugadores. En su turno el jugador pone la pieza, la pieza al menos debe tocar a otra pieza en juego.

4. Cada pieza usada en las ecuaciones vale un punto. Escribe la ecuación en la tarjeta de de puntajes de los taps hexagonal, incluyendo el puntaje total para cada ecuación. El jugador tiene 3 minutos para completar su turno.

5. El juego termina después de que no existe piezas en las manos de los jugadores.

6. El jugador con el puntaje más alto gana.

7.

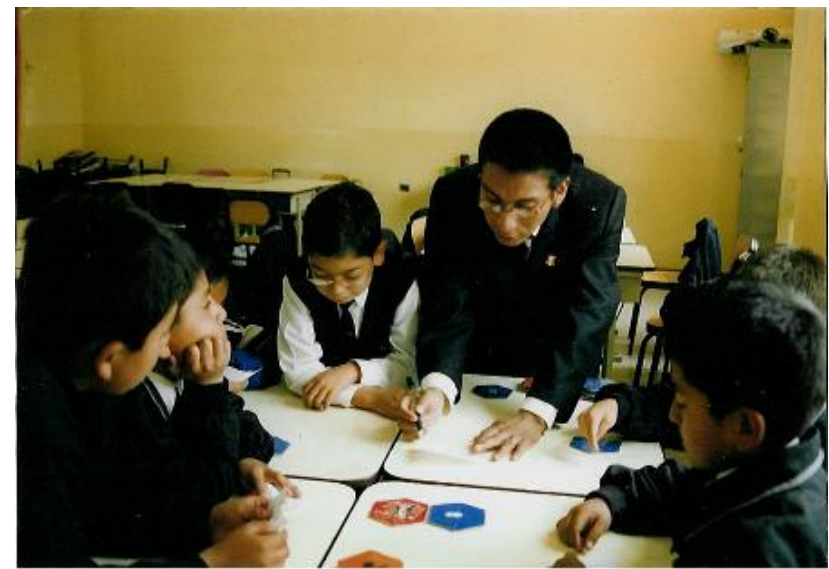

Figura 03. Alumnos escuchando las indicaciones del juego.

\section{Indicadores:}

Analiza los taps hexagonal y los caracteriza.

Seleccionar el o los criterios de ordenamiento (lógicos, cronológicos, etc).

Clasificar y ordenar estos rasgos.

Determinar los objetivos de la observación.

Operar y resuelve ejercicios mentalmente.

Identificar las habilidades del pensamiento durante el juego. 


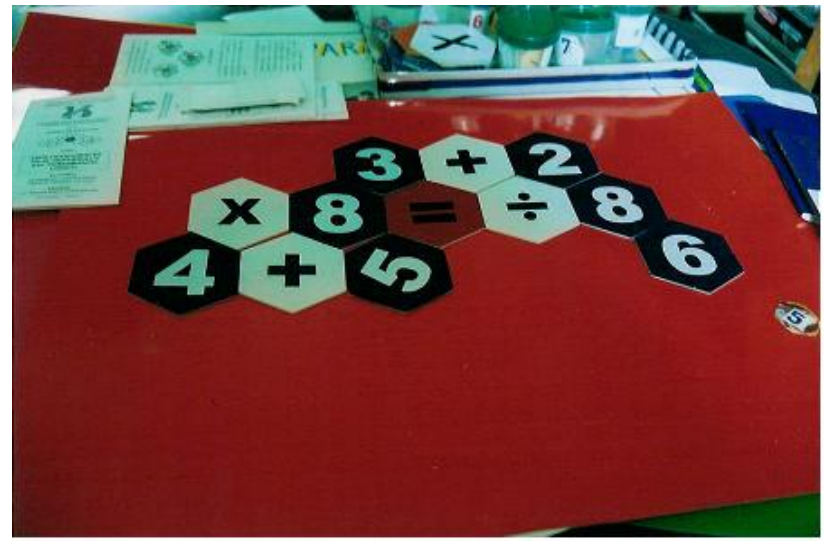

Figura 04. Ecuación creada por el alumno Christian Zárate Porras $68 \div 2+3=8 \times 4+5$, número de puntos es 12 .

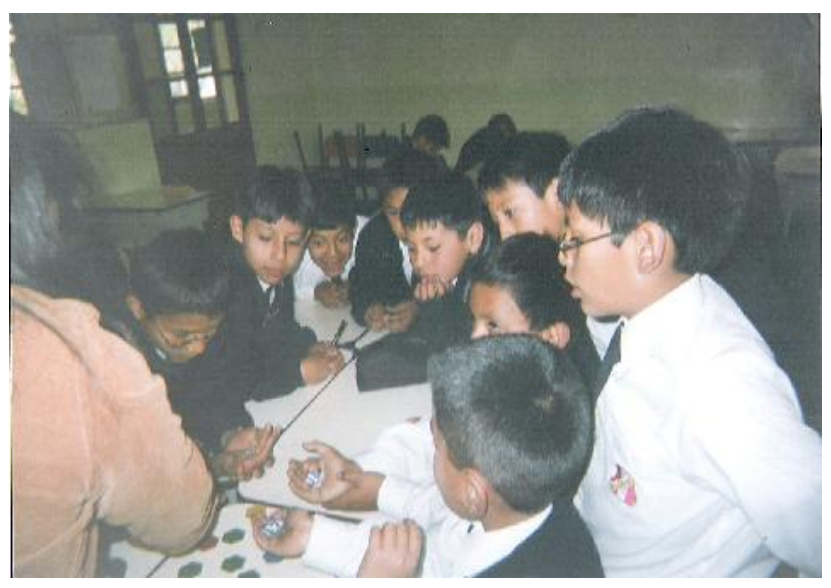

Figura 05. Alumnos del quinto grado trabajando en equipo los taps hexagonales.

\section{Habilidades del desarrollo del pensamiento lógico}

Observar

Identificar

Clasificar

Ordenar y

Generalizar

\section{CONCLUSIONES}

Al concluir la experimentación de los taps hexagonal en el desarrollo del pensamiento lógico en los alumnos del quinto fueron los siguientes:

- En forma general los taps hexagonal sistemáticamente diseñados y aplicados, influyen en contribuir a la solución de problema de ecuaciones en forma de juegos desarrollando la habilidad del pensamiento lógico identifica, clasificar, ordenar, generalizar y observar.

- En forma particular, los taps hexagonales en todos los resultados finales han influido significativamente en el desarrollo del pensamiento lógico en los alumnos del quinto grado "C" a comparación del quinto grado "B" con notas aprobatorias de "A", con más de un $85 \%$

\section{REFERENCIAS BIBLIOGRAFICAS}

1. BARAHONA, F. "Metodología de trabajos científicos" Editorial IPLER Bogotá.

2. GARCIA, J. La enseñanza Primario en el umbral del Siglo XIII. 1963 UNESCO 1988, pág. 120 a 229.

3. Morales, M. (2006). "Las fracciones según los pescantes". Revista Iberoamericana de Educación Matemática. 3 19.

4. MINISTERIO DE EDUCACIÓN, (2005) "Diseño Curricular Nacional de Educación Básica Regular, 122 a 129.

5. Nucleus Learning, Bogusia Gierus \& Alex Gierus

6. Piaget, J. (1974). Estructuralismo. Orbis.

7. Santiago, L. y Gonzalo, T. (2006). "Abejas y geometría”. Revista Iberoamericana de Educación Matemática. 139 -142 .

E-mail: irojasm5@hotmail.com vanslife9@yahoo.es 